\title{
Prevalence of osteo-renal impairment in the Romanian HIV cohort
}

\author{
Anca Streinu-Cercel ${ }^{1,2}$, Oana Săndulescu ${ }^{1,2^{*}}$, Gabriela Ceapraga ${ }^{2}$, Daniela Manolache², Monica Andreea Stoica ${ }^{2}$, \\ Liliana Lucia Preoțescu ${ }^{1,2}$ and Adrian Streinu-Cercel ${ }^{1,2}$
}

From The 11th Edition of the Scientific Days of the National Institute for Infectious Diseases "Prof. Dr. Matei Balș"' Bucharest, Romania. 14-16 October 2015

\begin{abstract}
Background: The Romanian HIV cohort has certain particularities that render it unique in Europe. We have performed a study to evaluate the prevalence of bone and kidney impairment in this particular group of HIVinfected patients.

Methods: We performed dual-energy X-ray absorptiometry (DXA) evaluation of the lumbar vertebrae and the femur, as well as laboratory tests including standard serum panels, bone-related markers and urinalysis in patients from the Romanian HIV cohort.

Results: The study included 72 patients, of which 46 (58.3\%) were males. The median (IQR) age was 38 (18) years and the median (IQR) time from HIV infection diagnosis was 9 (13) years. Most patients (55.6 \%) were non-smokers, but a relatively high proportion (37.5\%) was currently smoking. Only a small percentage of patients (20.8 \%) did not present any comorbidities, while $40.3 \%$ had one comorbidity, the most frequent being dyslipidemia (present in 25 patients, $38.5 \%$ ). Only 6 patients had a medical history suggestive for renal disease and 3 for bone-related abnormalities.

The median (IQR) glomerular filtration rate was 97.5 (33.0) mL/min/1.73sqm. We diagnosed 21 patients (29.6\%) with stage 2 chronic kidney disease and one patient (1.4\%) with stage 3 chronic kidney disease. Proteinuria was present in $9(12.7 \%)$ patients. The estimated glomerular filtration rate was significantly lower in patients with cardiac comorbidities $(p=0.013)$.

Vitamin D was significantly lower in smokers compared with non-smokers, with a mean value of $15 \mathrm{vs} .21 \mathrm{ng} / \mathrm{mL}$ and a moderate effect size (Cohen's $d=-0.5)(p=0.046)$. Lumbar osteopenia and osteoporosis were diagnosed in 33.3 and $13.7 \%$ of patients, while femoral osteopenia and osteoporosis were diagnosed in 37.3 and $7.8 \%$, respectively. Lower nadir CD4 cell counts were found in patients with bone-related comorbidities $(p=0.000)$.

Conclusions: We identified a relatively high prevalence of chronic kidney disease in the Romanian HIV cohort, and a fairly low prevalence of osteopenia and osteoporosis, compared with other European countries. In this category of patients smoking should be avoided altogether, as it may be an indirect risk factor for kidney disease (associating cardiac comorbidities) and it may impair bone metabolism by altering serum levels of hydroxy-vitamin D.
\end{abstract}

Keywords: Bone mineral density, DXA, eGFR, Chronic kidney disease, HIV

\footnotetext{
* Correspondence: oanasandulescu1@gmail.com

${ }^{1}$ Carol Davila University of Medicine and Pharmacy, Bucharest, Romania

${ }^{2}$ National Institute for Infectious Diseases "Prof. Dr. Matei Balș", Bucharest,

Romania
} 


\section{Background}

The Romanian HIV cohort has certain particularities that render it unique in Europe. The circulating HIV strains belong mainly to clade $\mathrm{F}$ and infection was acquired at an early age, in infancy and childhood, in the late '80s [1]. Therefore, patients from the Romanian cohort have seen antiretroviral (ARV) drugs come and go, and they have tried all available therapeutic options, even before the HAART era, when combination therapy had not yet been deemed as standard of care [2].

Given this unique background, and the patients' history of over 20 years of HIV infection [3], we performed a study to check for renal impairment and bone demineralization in patients monitored for HIV infection in one Romanian HIV reference center in Bucharest [4], in order to design individualized therapeutic approaches and updated guidelines for screening and management in this particular patient population $[5,6]$.

\section{Methods}

We performed a cross-sectional study in patients from the Romanian HIV cohort, to evaluate renal function through urinalysis, serum creatinine, serum urea, creatinine clearance and estimated glomerular filtration rate (eGFR), calculated using the Modification of Diet in Renal Disease (MDRD) formula [7]. Renal stiffness was assessed by one trained operator through shear-waves elastography on Aixplorer (SuperSonic Imagine, Aix-enProvence, France). The bone was evaluated through dual-energy X-ray absorptiometry (DXA) scan of the lumbar vertebrae and the left femur. Bone-related markers such as serum hydroxy-vitamin D and parathyroid hormone levels were also quantified. Serum calcium levels but not serum phosphorus levels were assessed.

The Romanian HIV cohort currently includes 13536 patients (data current through 30 September 2015) [8], from a total of 20968 patients diagnosed and monitored between 1985 and 2015. Of these, approximately 2200 are under active surveillance in the National Institute for Infectious Diseases "Prof. Dr. Matei Balș", Bucharest, and 72 patients presented for evaluation and accepted to participate in this study between January 2015 and August 2015.

Prior to inclusion in the study, all patients signed a written informed consent form. The study protocol was approved by the local Ethics Committee.

Statistical analysis was performed with SPSS Statistics for Windows (version 22.0, IBM Corp, Armonk, NY, USA). The descriptive analysis presents the mean and standard deviation (SD) for normally-distributed data or the median and interquartile range (IQR) values for non-normally-distributed data. The statistical analysis reports the independent-samples $t$ test with effect size calculation for normally-distributed variables, and the
Mann-Whitney U non-parametric test for independent samples, for non-normally-distributed variables. $P$ values were considered statistically significant when $<0.05$.

\section{Results}

The study included 72 patients, of which 46 (58.3\%) were males. The median (IQR) age was 38 (18) years and the median (IQR) time from HIV infection diagnosis was 9 (13) years. Patient characteristics are presented in Table 1. The mean \pm SD weight and height were $71 \pm$ $15.3 \mathrm{~kg}$ and $171 \pm 9.8 \mathrm{~cm}$, respectively, with a mean $\pm \mathrm{SD}$ body mass index of $23.7 \pm 3.7 \mathrm{~kg} / \mathrm{sqm}$. At the time of evaluation, most of the patients had received one prior ARV regimen (31.9\%) or over three prior regimens (30.6\%), while only 3 subjects were at their first treatment regimen (4.2\%). Most patients (54.2\%) had detectable and quantifiable HIV viral loads, while only $38.9 \%$ had undetectable HIV viral loads. The median (IQR) current CD4 cell count was 508 (380) cells $/ \mathrm{cmm}$, with a nadir count of 387 (290) cells/cmm, in the range of $19-1190$ cells/cmm.

Most patients (55.6\%) were non-smokers, but a relatively high proportion (37.5\%) was currently smoking. Only a small percentage of patients (20.8\%) did not present any comorbidities, while $40.3 \%$ had one comorbidity, the most frequent being dyslipidemia (present in 25 patients, $38.5 \%)$. Thirteen patients $(18.1 \%)$ had 2 comorbidities, 2 patients $(2.8 \%)$ displayed 3 comorbidities and 5 patients $(6.9 \%)$ had 4 comorbidities. Importantly, only 6 patients had a medical history suggestive for renal disease: renal cyst $(n=3)$, renal angiomyolipoma $(n=1)$, renal lithiasis $(n=2)$, and hydronephrosis $(n=1)$. Interestingly, of these 6 patients 4 had normal eGFR (and negative proteinuria), while the other two patients presented stage 1 and 3 chronic kidney disease, respectively. Three patients had positive medical history for bone-related abnormalities (herniated lumbar disc, bilateral coxarthrosis, and rheumatoid arthritis, respectively). Of these, two had insufficient serum levels of hydroxy-vitamin D, abnormal (high/low) parathyroid hormone levels but normal bone mineralization, while the third patient had normal serum levels of hydroxyvitamin $\mathrm{D}$ and parathyroid hormone but was diagnosed with femoral osteopenia.

One patient refused to undergo urinalysis or serum screening for kidney function. For the rest of the patients, the median (IQR) eGFR estimated by MDRD was 108.4 (34.7) $\mathrm{mL} / \mathrm{min} / 1.73 \mathrm{sqm}$ (Table 2). Based on our screening procedures, 21 patients $(29.6 \%)$ were diagnosed with stage 2 chronic kidney disease (with a mild decrease in eGFR, in the range of $60-89.9 \mathrm{~mL} / \mathrm{min} / 1.73 \mathrm{sqm}$ ) and one patient $(1.4 \%)$ with stage 3 chronic kidney disease (eGFR of $59.5 \mathrm{~mL} / \mathrm{min} / 1.73 \mathrm{sqm}$ ). Proteinuria was present in only $9(12.7 \%)$ of patients. 
Table 1 Patient characteristics

\begin{tabular}{|c|c|c|c|c|}
\hline Characteristic & Sub-category & $n(\%)$ & Median (IQR) & Percentiles 25,75 \\
\hline Male gender & & $46(58.3)$ & & \\
\hline Age, years & & & $38(18)$ & 26,44 \\
\hline Time since HIV diagnosis, years & & & $9(13)$ & 4,17 \\
\hline \multirow[t]{6}{*}{ Time since HIV diagnosis, years } & 1 & $10(13.9)$ & & \\
\hline & $2-5$ & $15(20.8)$ & & \\
\hline & $6-10$ & $13(18.1)$ & & \\
\hline & $11-15$ & $12(16.7)$ & & \\
\hline & $16-20$ & $14(19.4)$ & & \\
\hline & $21-27$ & $8(11.1)$ & & \\
\hline \multirow[t]{5}{*}{ Number of prior ARV regimens $(n=67)$} & 0 & $3(4.5)$ & & \\
\hline & 1 & $23(34.3)$ & & \\
\hline & 2 & $18(26.9)$ & & \\
\hline & 3 & $1(1.5)$ & & \\
\hline & $>3$ & $22(32.8)$ & & \\
\hline Current CD4 cell count, cells/cmm & & & $508(380)$ & 289,669 \\
\hline Nadir CD4 cell count, cells/cmm & & & $387(290)$ & 229,519 \\
\hline \multirow[t]{3}{*}{ Current viral load, copies/mL } & Undetectable & $28(38.9)$ & & \\
\hline & Below LLQ & $5(6.9)$ & & \\
\hline & Detectable (range: 27 to $170,000,000$ ) & $39(54.2)$ & 5617 (95224) & $37.5,95261$ \\
\hline \multirow[t]{4}{*}{ Smoking status } & Ex-smoker ${ }^{\mathrm{b}}$ & $2(2.8)$ & & \\
\hline & Current-smoker & $27(37.5)$ & & \\
\hline & Non-smoker & $40(55.6)$ & & \\
\hline & Refused to declare & $3(4.2)$ & & \\
\hline \multirow[t]{5}{*}{ Number of comorbidities ( $n=65$ ) } & 0 & $15(20.8)$ & & \\
\hline & 1 & $29(40.3)$ & & \\
\hline & 2 & $13(18.1)$ & & \\
\hline & 3 & $2(2.8)$ & & \\
\hline & 4 & $5(6.9)$ & & \\
\hline \multirow[t]{5}{*}{ Type of comorbidities $(n=65)$} & Cardiac & $5(7.7)$ & & \\
\hline & Metabolic & $28(43.1)$ & & \\
\hline & Renal & $6(9.2)$ & & \\
\hline & Bone-related & $3(4.6)$ & & \\
\hline & Hemato-oncologic & $5(7.7)$ & & \\
\hline
\end{tabular}

$A R V$ antiretroviral, IQR interquartile range, $L L Q$ lower limit of quantitation

aHIV-RNA TaqMan - limit of detection of 20 copies $/ \mathrm{mL}$

${ }^{b}$ Ex-smokers were defined as having completely quit smoking for over 6 months

The eGFR did not differ significantly with gender $(p=$ $0.523)$, smoking status $(p=0.109)$, number of comorbidities $(p=0.387)$, presence of bone-related comorbidities $(p=0.871)$, kidney $(p=0.290)$, metabolic $(p=0.160)$ or hemato-oncologic comorbidities $(p=0.541)$. However, eGFR was significantly lower in patients with cardiac comorbidities $(p=0.013, \mathrm{U}=52)$.

Bone-related markers were measured for 63 patients who consented to the blood tests. Hypoparathyroidism was diagnosed in $7.9 \%$ of patients, and hyperparathyroidism in
$33.3 \%$. Most patients (65.1\%) had insufficient serum levels of hydroxy-vitamin D, and $25.4 \%$ had hydroxy-vitamin D deficiency (Table 3). Notably, screening was performed during spring and summer. Vitamin D was significantly lower in smokers compared with non-smokers, with a mean value of $15 \mathrm{vs.} 21 \mathrm{ng} / \mathrm{mL}$ and a moderate effect size (Cohen's d $=-0.5)(p=0.046)$.

DXA scans were performed in 51 patients; the rest of the patients did not consent to the scan. Almost half $(45.1 \%)$ of the results were suggestive for normal 
Table 2 Kidney function tests results for patients screened in this study $(n=71)$

\begin{tabular}{|c|c|c|c|c|c|}
\hline Characteristic & Sub-category & $n(\%)$ & Median (IQR) & Percentiles 25, 75 & Mean \pm SD \\
\hline Serum urea, mg/dL & & & $\mathrm{N} / \mathrm{A}$ & N/A & $30.2 \pm 8.5$ \\
\hline Serum creatinine, mg/dL & & & $0.8(0.3)$ & $0.7,1.0$ & N/A \\
\hline eGFR, mL/min/1.73 sqm & & & $108.4(34.7)$ & $89.5,124.2$ & N/A \\
\hline \multirow[t]{5}{*}{ eGFR } & Stage $1>90$ & $49(69.0)$ & & & \\
\hline & Stage 2 60-89.9 & $21(29.6)$ & & & \\
\hline & Stage 3 30-59.9 & $1(1.4)$ & & & \\
\hline & Stage 4 15-29.9 & $0(0)$ & & & \\
\hline & Stage $5<15$ & $0(0)$ & & & \\
\hline Positive proteinuria, $n$ (\%) & & $9(12.7)$ & & & \\
\hline Renal elastography, kPa & & & $17.1(11)$ & $12.4,23.4$ & N/A \\
\hline
\end{tabular}

bone mineral density, but a high proportion of patients already displayed osteopenia (33.3\% with lumbar and $37.3 \%$ with femoral localization). Furthermore, lumbar osteoporosis was diagnosed in $13.7 \%$ of patients (with ages of 26, 30, 33, 38, 40, 47 and 65 years) and femoral osteoporosis in $7.8 \%$ of patients (with ages of 26, 40, 44, 50 years).

Lumbar or femoral T-scores did not differ significantly with gender ( $p=0.850$ and $p=0.961$, respectively), number of comorbidities $(p=0.168$ and $p=0.126$, respectively), presence of bone-related comorbidities $(p=0.933$ and $p=0.826$, respectively), kidney ( $p=0.489$ and $p=$ 0.750 , respectively), metabolic $(p=0.291$ and $p=0.394$, respectively), cardiac $(p=0.828$ and $p=0.343$, respectively) or hemato-oncologic comorbidities $(p=0.306$ and $p=0.618$, respectively).

We identified a statistical association between bonerelated comorbidities and nadir CD4 cell count, patients with this type of comorbidities having lower mean nadir CD4 cell counts (150 vs. 406 cells $/ \mathrm{cmm})$, with a large effect size (Cohen's $d=-1.5$ ). No other statistical associations were found between other types

Table 3 Bone-related tests results for patients screened in this study

\begin{tabular}{|c|c|c|c|c|c|}
\hline Characteristic & Sub-category & $n(\%)$ & Median (IQR) & Percentiles 25, 75 & Mean \pm SD \\
\hline \multirow[t]{5}{*}{ DXA scan result $(n=51)$} & Normal bone mineral density & $23(45.1)$ & & & \\
\hline & Lumbar osteopenia & $17(33.3)$ & & & \\
\hline & Lumbar osteoporosis & $7(13.7)$ & & & \\
\hline & Femoral osteopenia & $19(37.3)$ & & & \\
\hline & Femoral osteoporosis & $4(7.8)$ & & & \\
\hline Lumbar L1-L4 DXA T-score & & & $\mathrm{N} / \mathrm{A}$ & $\mathrm{N} / \mathrm{A}$ & $-0.8 \pm 1.0$ \\
\hline Left femur DXA T-score & & & $\mathrm{N} / \mathrm{A}$ & $\mathrm{N} / \mathrm{A}$ & $-0.4 \pm 0.8$ \\
\hline Serum parathyroid hormone, pg/mL & & & $48.2(43.6)$ & $27.7,71.3$ & $\mathrm{~N} / \mathrm{A}$ \\
\hline \multirow[t]{3}{*}{ Interpretation of serum parathyroid hormone $(n=63)$} & Decreased $(<15$ pg/mL) & $5(7.9)$ & & & \\
\hline & Normal (15-65 pg/mL) & $37(58.7)$ & & & \\
\hline & Increased (>65 pg/mL) & $21(33.3)$ & & & \\
\hline Serum hydroxy-vitamin $D, n g / m L$ & & & $\mathrm{~N} / \mathrm{A}$ & $\mathrm{N} / \mathrm{A}$ & $18.8 \pm 10.8$ \\
\hline \multirow[t]{4}{*}{ Interpretation of serum hydroxy-vitamin D levels $(n=63)$} & Deficient $(<10$ ng/mL) & $16(25.4)$ & & & \\
\hline & Insufficient (10-29.9 ng/mL) & $41(65.1)$ & & & \\
\hline & Optimal (30-99.9 ng/mL) & $6(9.5)$ & & & \\
\hline & Toxic (>100 ng/mL) & $0(0)$ & & & \\
\hline
\end{tabular}


Table 4 Statistical associations between type of comorbidities and laboratory parameters

\begin{tabular}{llllllll}
\hline $\begin{array}{l}\text { Type of } \\
\text { comorbidities }\end{array}$ & $\begin{array}{l}\text { Current CD4 cell } \\
\text { count }\end{array}$ & $\begin{array}{l}\text { Nadir CD4 cell } \\
\text { count }\end{array}$ & $\begin{array}{l}\text { HIV viral } \\
\text { load }\end{array}$ & $\begin{array}{l}\text { Renal } \\
\text { stiffness }\end{array}$ & $\begin{array}{l}\text { Hydroxy-vitamin } \\
\text { D }\end{array}$ & $\begin{array}{l}\text { Parathyroid } \\
\text { hormone }\end{array}$ & $\begin{array}{l}\text { Smoking } \\
\text { status }\end{array}$ \\
\hline Cardiac & 0.472 & 0.685 & 0.321 & 0.443 & 0.910 & 0.053 & 0.052 \\
Metabolic & 0.136 & 0.774 & 0.326 & 0.553 & 0.579 & 0.091 & 0.090 \\
Renal & 0.275 & 0.362 & 0.321 & N/A & 0.365 & 0.532 & 1.000 \\
Bone-related & 0.969 & 0.000 & 0.326 & 0.443 & 0.500 & 0.613 & 0.324 \\
Hemato-oncologic & 0.551 & 0.818 & 0.321 & 0.773 & 0.601 & 0.695 & 1.000 \\
\hline
\end{tabular}

$P$ values are reported. Statistically-significant results are marked in bold

Renal stiffness was assessed for 19 patients by one trained operator through shear-waves elastography on Aixplorer (SuperSonic Imagine, Aix-en-Provence, France)

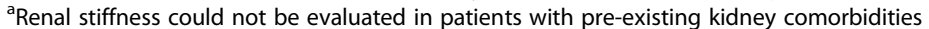

of comorbidities and current or nadir CD4 cell count, HIV viral load, renal stiffness, hydroxy-vitamin $D$ or parathyroid hormone (Table 4).

\section{Discussion}

Our study has described the epidemiology of chronic kidney disease and bone demineralization in HIVpositive patients from the Romanian HIV cohort. In this study $29.6 \%$ of patients were diagnosed with stage 2 kidney disease and $1.4 \%$ with stage 3 kidney disease. These numbers are quite high, compared with a prevalence of chronic kidney disease in patients with HIV infection reported at $6 \%$ in the Danish HIV cohort study [9], 24\% in Nigeria [10], and $2 \%$ [11], $10.2 \%$ [12] or $15.5 \%$ [13] in the United States of America.

We have also identified that cardiac comorbidities are risk factors for kidney disease in HIV-positive patients from our cohort $(p=0.013)$. Other similar studies also report hypertension as a risk factor for additional kidney damage in HIV-positive patients [11] compared with the HIV-negative population. Smoking has not been identified as a direct risk factor for renal disease $(p=0.109)$, our results being in line with data reported from other European countries, for example the Danish HIV cohort study [9]; however, given the recognized correlation between smoking and cardiovascular disease and our study's findings that cardiac comorbidities associate a decreased eGFR, we suggest that HIV-positive patients who are current smokers should also be monitored for renal function. Moreover, as our study has shown that smoking is associated with significantly lower levels of hydroxy-vitamin $\mathrm{D}$, this points to the fact that smoking should be avoided altogether by HIVpositive patients, as it not only targets the kidney, but the bone metabolism as well.

Lumbar osteopenia and osteoporosis were diagnosed in 33.3 and $13.7 \%$ of patients, respectively; femoral osteopenia and osteoporosis were diagnosed in 37.3 and $7.8 \%$, respectively. Notably, the median age in this study was 38 years (IQR: 18 years), suggesting that this high prevalence of bone and kidney disease is rather correlated with the long evolution of HIV infection (median: 9 years, IQR: 13 years) than with patient age. These results are, to an extent, encouraging, as other studies report a higher prevalence of osteopenia or osteoporosis in HIV-positive patients, i.e., 51 and $10 \%$, respectively, in the United States of America [14], 63.2 and 15.1\% in Italy [15], and 56.5 and $10.7 \%$ in Spain [16]. However, studies from Brazil report a much lower prevalence of decreased bone mineral density in patients with HIV infection, i.e. $14.6 \%$ in the lumbar vertebrae and $5.6 \%$ in the femur [17].

We also found lower nadir CD4 cell counts in patients with bone-related comorbidities $(p=0.000)$. A recent study from Australia defined a series of risk factors for low bone mineral density in treatment-naïve HIVpositive patients, and they found no association with nadir CD4 cell count [18]. However, seeing as that study was performed in patients with mean nadir CD4 cell counts above 500 cells/cmm, who had not yet met any criteria for starting ARV treatment, we expect their data to be significantly different from that derived from our study, where most patients were under ARV treatment, and the nadir CD4 cell count was 387 cells/cmm. Our finding that lower nadir CD4 cell counts are associated with bone-related abnormalities confirm previous studies performed in Spain, which reported lower bone mineral density in patients with lower nadir CD4 cell count [16].

\section{Conclusions}

We identified a relatively high prevalence of chronic kidney disease in the Romanian HIV cohort, and a fairly low prevalence of osteopenia and osteoporosis, compared with other European countries. Lower nadir CD4 cell counts were statistically correlated with the presence of bone-related comorbidities. In this category of patients smoking should be avoided altogether, as it may be an indirect risk factor for kidney disease (associating cardiac comorbidities) and it may impair bone metabolism by altering serum levels of hydroxy-vitamin $\mathrm{D}$. 


\section{Abbreviations}

ARV: antiretroviral; DXA: dual-energy X-ray absorptiometry; eGFR: estimated glomerular filtration rate; HAART: highly active antiretroviral therapy; IQR: interquartile range; SD: standard deviation.

\section{Competing interests}

The authors declare that they have no competing interests.

\section{Authors' contributions}

ASC, OS and Adrian SC contributed to study design. ASC, OS, GC, DM, MAS and LLP contributed to acquisition and analysis of data. ASC and OS performed the statistical analysis. ASC, OS, LLP and Adrian SC contributed to interpretation of data. OS drafted the manuscript. ASC, OS, GC, DM, MAS, LLP and Adrian SC revised the manuscript critically for important intellectual content. All authors approved the final version of the manuscript.

\section{Acknowledgements}

1) Partially supported by the Osteo Renal Program, AbbVie.

2) Partially supported by the Sectoral Operational Programme Human

Resources Development (SOP HRD), financed from the European Social Fund and by the Romanian Government under the contract numbers POSDRU/ 159/1.5/S/137390

None of the supporting bodies were involved in study design, data

collection, data interpretation, or manuscript preparation.

\section{Declaration}

Publication of this article was funded by the Prof. Dr. Matei Balș Foundation, Bucharest, Romania.

\section{Published: 17 March 2016}

\section{References}

1. Manolescu LS, Temereanca A, Ruta S. HIV-1 circulating subtypes in Romania. Roum Arch Microbiol Immunol. 2013;72:121-34

2. Streinu-Cercel A, Săndulescu O, Streinu-Cercel A. Sudden-onset kidney impairment in an HIV-positive patient. J Contemp Clin Pract. 2015;1:19-21. doi:10.18683/jccp.2015.1004.

3. Aramă V, Tilișcan C, Ion D, Mihăilescu R, Munteanu D, Streinu-Cercel A, et al. Serum adipokines and HIV viral replication in patients undergoing antiretroviral therapy. Germs. 2012;2:12-7. doi:10.11599/germs.2012.1008.

4. Streinu-Cercel A, Săndulescu O, Șchiopu C, Oprea C, Rugină S, Dorobăț C, et al. Screening for osteo-renal involvement in the Romanian HIV cohort. The 10th Edition of the Scientific Days of the National Institute for Infectious Diseases "Prof Dr Matei Bals"; 2014; Bucharest, Romania. BMC Infect Dis. 2014;14 Suppl 7:P65.

5. Streinu-Cercel A. HIV and bone mineral density. Germs. 2015;5:7. doi:10. 11599/germs.2015.1064.

6. Streinu-Cercel A. Osteo-renal impairment in HIV infection. Germs. 2014;4:29. doi:10.11599/germs.2014.1052.

7. Levey AS, Bosch JP, Lewis JB, Greene T, Rogers N, Roth D. A more accurate method to estimate glomerular filtration rate from serum creatinine: a new prediction equation. Modification of Diet in Renal Disease Study Group. Ann Intern Med. 1999;130:461-70. doi:199903160-00002 [pii].

8. Romanian National Committee for Fighting Against AIDS. HIV/AIDS infection in Romania - update 1st December 2015. 2015. http://cnlas.ro/images/doc/ 01122015 rom.pdf. Accessed: 28 Nov 2015.

9. Ahlstrom MG, Feldt-Rasmussen B, Legarth R, Kronborg G, Pedersen C, Larsen CS, et al. Smoking and renal function in people living with human immunodeficiency virus: a Danish nationwide cohort study. Clin Epidemiol. 2015;7:391-9. doi:10.2147/CLEP.S83530. clep-7-391.

10. Adedeji TA, Adedeji NO, Adebisi SA, Idowu AA, Fawale MB, Jimoh KA. Prevalence and Pattern of Chronic Kidney Disease in Antiretroviral-Naive Patients with HIV/AIDS. J Int Assoc Provid AIDS Care. 2015;14:434-40. doi:10. 1177/2325957415587570

11. Gupta SK, Mamlin BW, Johnson CS, Dollins MD, Topf JM, Dube MP. Prevalence of proteinuria and the development of chronic kidney disease in HIV-infected patients. Clin Nephrol. 2004;61:1-6.

12. Fulop T, Olivier J, Meador RS, Hall J, Islam N, Mena L, et al. Screening for chronic kidney disease in the ambulatory HIV population. Clin Nephrol. 2010;73:190-6. doi:7338 [pii].
13. Wyatt CM, Winston JA, Malvestutto CD, Fishbein DA, Barash I, Cohen AJ, et al. Chronic kidney disease in HIV infection: an urban epidemic. AIDS. 2007;21: 2101-3. doi:10.1097/QAD.0b013e3282ef1bb4. 00002030-200710010-00013.

14. Escota GV, Mondy K, Bush T, Conley L, Brooks JT, Onen N, et al. High prevalence of low bone mineral density and substantial bone loss over 4 years among HIV-infected persons in the era of modern antiretroviral therapy. AIDS Res Hum Retroviruses. 2015. doi:10.1089/aid.2015.0158.

15. Santi D, Madeo B, Carli F, Zona S, Brigante G, Vescini F, et al. Serum total estradiol, but not testosterone is associated with reduced bone mineral density (BMD) in HIV-infected men: a cross-sectional, observational study. Osteoporos Int. 2015. doi:10.1007/s00198-015-3383-8.

16. Negredo E, Domingo P, Ferrer E, Estrada V, Curran A, Navarro A, et al. Peak bone mass in young HIV-infected patients compared with healthy controls. J Acquir Immune Defic Syndr. 2014;65:207-12. doi:10.1097/01.qai. 0000435598.20104.d6.

17. Gomes DC, Valadares AL, Amaral E, de Oliveira FN, Pinto-Neto AM, Baccaro LF, et al. Association between HIV infection and bone mineral density in climacteric women. Arch Osteoporos. 2015;10:33. doi:10.1007/s11657-015-0238-z

18. Carr A, Grund B, Neuhaus J, Schwartz A, Bernardino Jl, White D, et al. Prevalence of and risk factors for low bone mineral density in untreated HIV infection: a substudy of the INSIGHT Strategic Timing of AntiRetroviral Treatment (START) trial. HIV Med. 2015;16 Suppl 1:137-46. doi:10.1111/hiv. 12242.

\section{Submit your next manuscript to BioMed Central and we will help you at every step:}

- We accept pre-submission inquiries

- Our selector tool helps you to find the most relevant journal

- We provide round the clock customer support

- Convenient online submission

- Thorough peer review

- Inclusion in PubMed and all major indexing services

- Maximum visibility for your research

Submit your manuscript at www.biomedcentral.com/submit 